\title{
Magnetostrictive properties of amorphous SmCo thin films with imprinted anisotropy
}

\author{
F. Magnus, * R. Moubah, V. Kapaklis, G. Andersson, and B. Hjörvarsson \\ Department of Physics and Astronomy, Uppsala University, Box 516, 75120 Uppsala, Sweden
}

(Received 18 February 2014; published 17 April 2014)

\begin{abstract}
We examine the magnetostriction in amorphous SmCo thin films with a composition in the range 4-27 at. \% Sm. The magnetostriction increases significantly with increasing Sm content but is small compared to terbium-based ferromagnetic compounds, despite the large imprinted anisotropy. The magnetostriction and anisotropy both increase approximately linearly as the temperature is reduced. The magnetoelastic energy is found to be far smaller than the anisotropy energy so the magnetoelastic atomic displacements during growth cannot be the origin of the imprinted anisotropy. The anisotropy is only slightly altered by the application of large tensile stresses, indicating that the local strain fields involved in magnetostriction are not equivalent to the global strain produced by mechanical bending.
\end{abstract}

DOI: 10.1103/PhysRevB.89.134414

PACS number(s): 61.43.Dq, 75.80.+q, 75.30.Gw

\section{INTRODUCTION}

Amorphous magnetic films are highly interesting from a fundamental science and technological point of view. Amorphous layers are uniform and smooth due to the absence of atomic steps, defects, and strain, which makes them particularly well suited for layered structures [1,2]. Their magnetic properties are highly tuneable [3] as compositions can be varied over a large range without having to consider changes in lattice constants. In addition, it is possible to imprint an in-plane uniaxial anisotropy in amorphous layers, in an arbitrary direction, by applying a magnetic field during growth $[4,5]$.

Despite the widespread use of field induced magnetic anisotropy, its origins in amorphous films are still a matter of debate. Although there is no long-range crystalline order in amorphous materials, the presence of magnetic anisotropy implies some directional preference in the short-range chemical or structural order brought about by the direction of the applied growth field. Short- to medium-range order has been shown to be present in amorphous materials but the precise form of order will depend strongly on the composition [6,7]. Several different types of ordering have been suggested as the source of magnetic anisotropy, such as an alignment of atomic moment pairs via dipolar effects [8], alignment of atomic clusters via local spin-orbit coupling (single-ion anisotropy) [9], and direction dependent bonding between atoms of different elements [10]. Imprinted anisotropy has also been linked with the strain induced during growth through magnetoelastic coupling [9].

The magnetoelastic coupling gives rise to magnetostriction, which macroscopically is manifest in the contraction or expansion of a material as its magnetization is rotated. Magnetostriction can be utilized in a variety of microelectromechanical systems $[11,12]$ but it can also be undesirable $[13,14]$. A large magnetostriction is generally found in materials which exhibit a high magnetic anisotropy. The largest effects are observed in crystalline TbDyFe compounds which have magnetostriction coefficients approaching $\lambda_{\mathrm{s}}=2000 \times 10^{-6}$ at room temperature [15]. Amorphous films of the same

*fridrik.magnus@physics.uu.se material exhibit lower magnetostriction, albeit at lower fields due to a reduced anisotropy [16,17]. Other high-anisotropy materials [18] such as crystalline SmCo also have a large magnetostriction although it is strongly dependent on the grain size [19]. It has been shown that a large anisotropy can even be induced in amorphous alloys of Sm and Co and it is tuneable over a large range through composition [20-23]. However, little is known about the magnetostriction in amorphous $\mathrm{SmCo}$.

Here we study the magnetostriction and inverse magnetostriction in amorphous SmCo films with a magnetic-fieldimprinted uniaxial in-plane anisotropy. We show that the magnetostriction can be tuned by means of the composition, independently of the anisotropy. We use the compositional and temperature dependence of the magnetostriction to gain an insight into the relationship between magnetostriction and the imprinted anisotropy, which we find to be a result of the local atomic configuration. We also determine the effect of mechanically applied stress on the anisotropy and discuss the importance of local versus global strain for the inverse magnetostriction effect.

\section{EXPERIMENT}

The samples were grown by dc magnetron sputtering onto 0.15 -mm-thick $\mathrm{Si}(100)$ substrates, with an area of $10 \times$ $10 \mathrm{~mm}^{2}$. A 2-nm-thick buffer layer of $\mathrm{Al}_{0.7} \mathrm{Zr}_{0.3}$ was deposited on the substrate before a 200-nm-thick SmCo alloy film, with a composition varying from 4-27 at. \% Sm, was grown by cosputtering from elemental targets of $\mathrm{Co}$ and $\mathrm{Sm}$. The buffer layer promotes the amorphous growth of the SmCo [2]. Finally, a 3-nm-thick capping layer of AlZr was grown to protect the SmCo layer from oxidation. All films were grown at room temperature, without any substrate cooling. A magnetic field of $0.1 \mathrm{~T}$ was applied during growth, using a sample holder equipped with two permanent magnets, as described in [4]. The magnetic field induces a well-defined uniaxial in-plane anisotropy, as confirmed by performing magneto-optic Kerr effect (MOKE) measurements (with $s$-polarized light) while rotating the sample about the azimuthal angle $\phi$. Details about the growth, structural properties, and magnetic anisotropy of these films can be found elsewhere [20].

The magnetostriction of the films was determined by measuring the sample curvature in an applied magnetic field. 
(a)

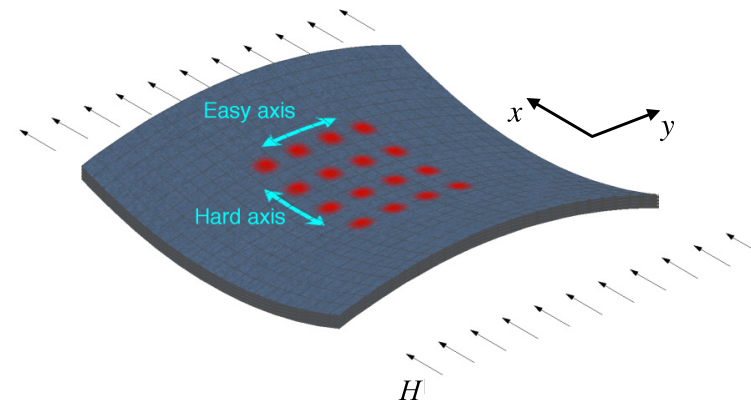

(b)

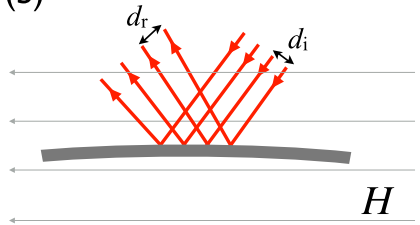

(c)

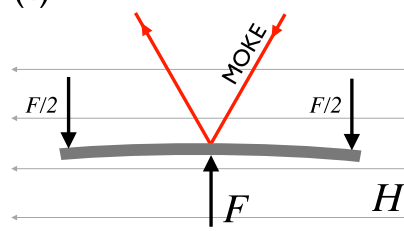

FIG. 1. (Color online) (a) The relationship between magnetostriction and curvature. An applied field $H$, parallel to the hard axis, induces a biaxial strain which results in a biaxial curvature. The grid of laser beams, used to measure the curvature, is aligned parallel to the hard and easy axes ( $x$ and $y$, respectively). (b) The grid of laser beams reflected off the surface of a curved sample in a magnetic field $H$. The spacing of the reflected beams $d_{\mathrm{r}}$ differs from the spacing of the incident beams $d_{\mathrm{i}}$, by an amount dependent on the curvature. (c) The MOKE laser reflected off a sample, curved by the applied force $F$, in an applied field $H$.

As the film is mechanically coupled to the substrate, it experiences a clamping when a magnetic field is applied. The induced stress therefore results in a bending of the sample as a whole, as shown in Fig. 1(a). The curvature is measured by reflecting a rectangular array of laser beams off the sample surface onto a CCD camera. The rectangular $x-y$ coordinate system of the array is aligned parallel to the easy and hard axes of the magnetic film and the incident beams are spaced in the $x$ and $y$ directions by a distance $d_{\mathrm{i}(x, y)}$. The curvature can be determined by the difference in spacing between the incident and reflected beams, $d_{\mathrm{r}(x, y)}-d_{\mathrm{i}(x, y)}$ [see Fig. 1(b)], along the $x$ and $y$ directions simultaneously. Taking the curvature at zero applied field as the reference point we measure the change in curvature as an external magnetic field is applied. The magnetic field is swept linearly from $\mu_{0} H_{\max }=360 \mathrm{mT}$ to $-\mu_{0} H_{\max }$ and back up to $\mu_{0} H_{\max }$ so that a full field cycle is performed. The relative curvature along each direction $\kappa_{x, y}$ can then be related to the film stress $\sigma_{x, y}$ through

$$
\sigma_{x, y}=\frac{1}{6} \frac{Y_{\mathrm{s}}}{1+v_{\mathrm{s}}} \frac{t_{\mathrm{s}}^{2}}{t_{\mathrm{f}}} \kappa_{x, y},
$$

where $Y_{\mathrm{s}}, v_{\mathrm{s}}$, and $t_{\mathrm{s}}$ are the Young modulus, Poisson ratio, and thickness of the substrate and $t_{\mathrm{f}}$ is the film thickness [24-26]. Young's modulus and Poisson's ratio in silicon are dependent on the orientation of the stress relative to the crystal axes of the sample. We assume that the stress is parallel to the hard magnetic axis of the SmCo which is aligned to the [100] direction of the Si substrate. Therefore, we use the values $Y_{\mathrm{s}}=130 \mathrm{GPa}$ and $v_{\mathrm{s}}=0.28$ [27].

To examine the effects of strain on anisotropy the samples were mechanically curved (bent) while measuring the magnetization using MOKE. The curvature was induced by applying a force with a micrometer screw to the back of the samples along a central axis parallel to one of the sides of the sample, as shown in Fig. 1(c). The sample edges were clamped from the top. This induces a uniaxial strain in the direction perpendicular to the axis along which the force is applied.

\section{RESULTS AND DISCUSSION}

In a magnetic film with uniaxial anisotropy, the magnetization will be aligned to the easy axis in the absence of an applied magnetic field. Magnetization of the film along the easy axis takes place by the propagation of $180^{\circ}$ domain walls so the magnetization is always parallel to the easy axis, except within the domain walls themselves. As the magnetostriction is an even function of magnetization direction, the application of a magnetic field along the easy axis does not result in an induced stress. In contrast, applying a magnetic field perpendicular to the easy axis rotates the magnetization of the SmCo film away from the easy axis which induces a stress, as seen in Fig. 2 for three different SmCo compositions. The sign of the stress is negative in the direction parallel to the applied field (the hard axis direction) corresponding to a film contraction [Fig. 2(a)]. The contraction in the hard axis direction results in an expansion in the easy axis direction as evidenced by the positive sign of the measured stress in Fig. 2(b). The resulting

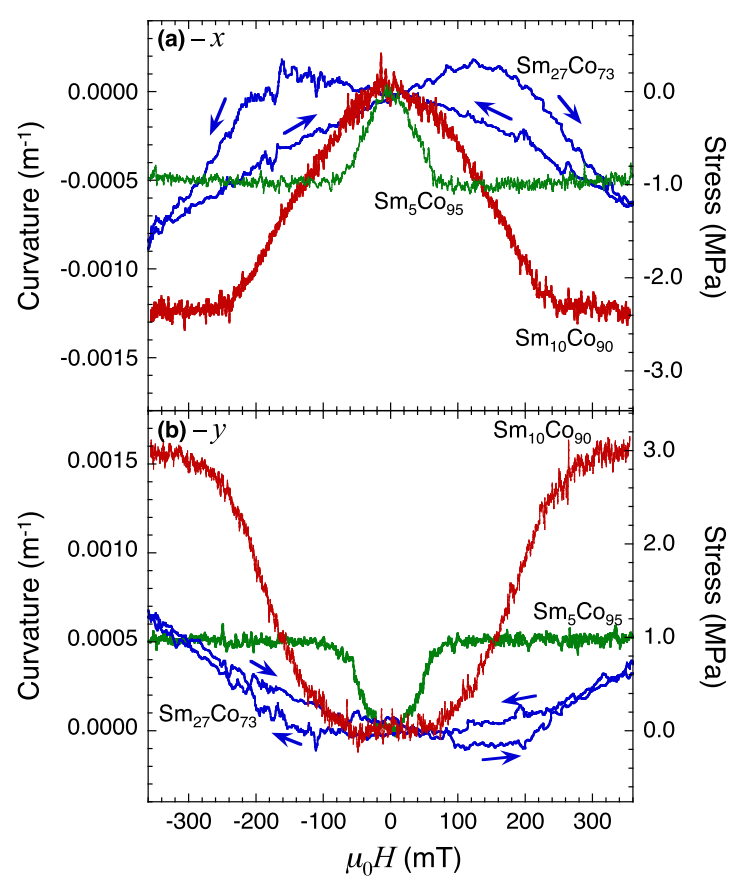

FIG. 2. (Color online) The curvature and stress of the $\mathrm{SmCo} / \mathrm{Si}$ samples as a function of applied magnetic field parallel to the hard axis (the $x$ direction). The stress at zero applied field is defined as zero. The curvature is measured simultaneously along two perpendicular directions: (a) parallel to the hard axis and (b) parallel to the easy axis. The three different SmCo compositions are shown and the arrows indicate the field sweep direction for the $\mathrm{Sm}_{27} \mathrm{Co}_{73}$ sample. The size of the magnetostriction is strongly dependent on the Sm content of the film. 
saddlelike shape of the sample is illustrated schematically in Fig. 1(a). The magnetostriction continues to increase with field as the film magnetization is rotated until the saturation field $H_{\text {sat }}$ is reached. The saturation field increases with increasing $\mathrm{Sm}$ content and is $680 \mathrm{mT}$ at room temperature for the $\mathrm{Sm}_{27} \mathrm{Co}_{73}$ sample [20], which exceeds the maximum field that can be applied in the curvature measurement setup.

If the magnetization takes place entirely by rotation the magnetostriction will follow [8]

$$
\frac{\lambda(H)}{\lambda_{\mathrm{s}}}=\left(\frac{M(H)}{M_{\mathrm{s}}}\right)^{2},
$$

where $\lambda$ and $M$ are the field dependent magnetostriction and magnetization, respectively, and the field $H$ is applied along the hard axis (the subscript $s$ refers to the values at saturation). In the case where part of the saturation magnetization is reached via $180^{\circ}$ domain wall motion, the magnetostriction remains constant initially and then increases as rotation takes over $[8,16,28]$. In our samples we have $180^{\circ}$ domain wall motion as well as rotation at low fields $[29,30]$ and therefore some flattening of the magnetostriction is expected at low fields. Figure 2 shows that such flattening is present although only along the easy axis direction. This becomes more apparent in the high Sm content samples with the larger anisotropy and magnetostriction. Such an anisotropic magnetostriction in the film plane is surprising as one would expect the two perpendicular in-plane directions to mimic each other due to volume conservation arguments. Another feature observed is the magnetostrictive hysteresis which appears in the $\mathrm{Sm}_{27} \mathrm{Co}_{73}$ film. This has also been observed previously in sintered $\mathrm{SmCo}_{5}$ bulk magnets [19]. The turnaround in the field induced stress in this sample is not at zero field but occurs in the 100-150-mT range which is similar to the coercive field in this film. This magnetostrictive hysteresis is the result of a hysteretic magnetization response [31] as we are performing minor loops of the magnetization.

The parameter used to describe the size of the magnetostriction in thin films is the magnetoelastic coupling coefficient, which is defined as the difference of the magnetic field induced stress for the field applied parallel and perpendicular to the easy axis, or [24,25]

$$
b^{\gamma, 2}=\sigma\left(H_{\|}\right)-\sigma\left(H_{\perp}\right) \text { at } H_{\|}, H_{\perp}>H_{\text {sat }},
$$

where $\sigma$ is found from Eq. (1). We find that $\sigma\left(H_{\|}\right)=0$ as expected and therefore $b^{\gamma, 2}$ is equal in magnitude to the saturation stress but of opposite sign. The magnetostriction coefficient $\lambda_{\mathrm{s}}$ can be estimated by

$$
\lambda_{\mathrm{s}}=-\frac{3}{2} \frac{1+v_{f}}{Y_{f}} b^{\gamma, 2},
$$

where $Y_{f}$ and $v_{f}$ are the Young modulus and Poisson ratio of the film. Unfortunately, these parameters cannot be determined accurately for thin films but we use $Y_{f}=120 \mathrm{GPa}$ and $v_{f}=$ 0.27 (obtained from data on commercial SmCo magnets) to get a rough estimate of $\lambda_{s}$. The results are shown in Table I. For the $\mathrm{Sm}_{27} \mathrm{Co}_{73}$ film we are unable to saturate the magnetostriction and therefore we can only estimate the coupling coefficient by extrapolating to the known saturation field (determined by MOKE magnetometry [20]).
TABLE I. The room-temperature magnetoelastic coupling coefficient $b^{\gamma, 2}$, magnetostriction constant $\lambda_{s}$, and the in-plane anisotropy constant $K_{\text {ip }}$ (from [20]) for three compositions $\mathrm{Sm}_{x} \mathrm{Co}_{100-x}$.

\begin{tabular}{lccc}
\hline \hline$x($ at. \% Sm) & $b^{\gamma, 2}(\mathrm{MPa})$ & $\lambda_{\mathrm{s}}$ & $K_{\text {ip }}\left(10^{5} \mathrm{~J} / \mathrm{m}^{3}\right)$ \\
\hline 4 & $1.01 \pm 0.06$ & $-16 \times 10^{-6}$ & $0.5 \pm 0.1$ \\
10 & $2.33 \pm 0.06$ & $-37 \times 10^{-6}$ & $1.6 \pm 0.2$ \\
27 & $3.2 \pm 0.8$ & $-51 \times 10^{-6}$ & $1.5 \pm 0.3$ \\
\hline \hline
\end{tabular}

The magnetostriction clearly increases with increasing Sm content. As described elsewhere [20], the in-plane anisotropy constant of amorphous SmCo thin films increases with increasing $\mathrm{Sm}$ content but peaks at a composition of approximately $\mathrm{SmCO}_{5}$, after which it decreases. The anisotropy constant for the compositions used here is shown in Table I. The anisotropy constant is similar for $\mathrm{Sm}_{10} \mathrm{Co}_{90}$ and $\mathrm{Sm}_{27} \mathrm{Co}_{73}$ and therefore the composition dependences of the anisotropy and magnetostriction are not the same. The sign of $b^{\gamma, 2}$ is positive, indicating a contraction in the direction parallel to the applied field. This is consistent with a previous study on magnetostriction in single-crystal and sintered $\mathrm{SmCo}_{5}$ which finds a contraction in the direction perpendicular to the $c$ axis (the $c$ axis is the easy axis) when the field is applied in the same direction [19]. However, the size of the magnetostriction coefficient is an order of magnitude smaller in our case compared to the values of $-760 \times 10^{-6}$ and $-160 \times 10^{-6}$ found for single-crystal and sintered $\mathrm{SmCo}_{5}$, respectively. The difference is due to the randomness in the atomic configuration of the amorphous films, which results in a suppression of the magnetoelastic interactions compared to crystalline films. The magnetostriction is also significantly smaller than in amorphous TbDyFe and TbCo alloys, where $\lambda_{\mathrm{s}}$ is in the $(400-1000) \times 10^{-6}$ range $[16,32]$.

The temperature dependence of the magnetostriction in the $\mathrm{Sm}_{4} \mathrm{Co}_{96}$ sample can be seen in Fig. 3(a). The shape of the magnetic-field dependence is the same at all temperatures studied but the magnetoelastic coupling coefficient increases as the temperature is reduced. The saturation field, defined from the intersection of two straight lines fitted to the saturation region and the linear magnetostrictive region, also increases with decreasing temperature. The temperature variation of $b^{\gamma, 2}$ and $H_{\text {sat }}$ is plotted in Fig. 3(b). Both parameters change approximately linearly with temperature, with the magnetoelastic coupling coefficient changing by $-0.006 \mathrm{MPa} / \mathrm{K}$. The saturation field is proportional to the uniaxial in-plane anisotropy constant $K_{\mathrm{ip}}$ and inversely proportional to the saturation magnetization $M_{\mathrm{s}}$, i.e.,

$$
H_{\mathrm{sat}}=\frac{2 K_{\mathrm{ip}}}{\mu_{0} M_{\mathrm{s}}} .
$$

The saturation magnetization of SmCo is almost constant in this temperature range [20] and therefore the increase in the saturation field is due to an increase in the anisotropy. From Eq. (5) and the linear fit in Fig. 3 we find that the change in anisotropy with temperature is approximately $-110 \mathrm{~J} / \mathrm{m}^{3} \mathrm{~K}$.

The similarity between the temperature dependence of $b^{\gamma, 2}$ and $H_{\text {sat }}$ raises the question whether the imprinted anisotropy could be a result of magnetoelastic effects during growth. 

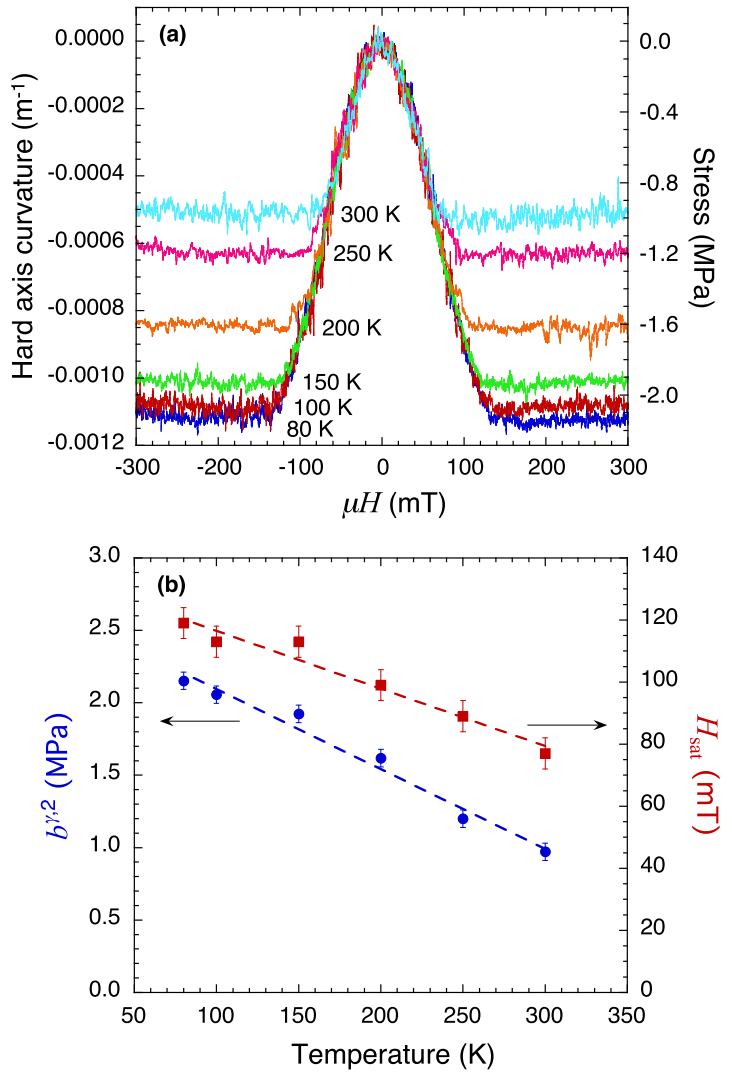

FIG. 3. (Color online) (a) The curvature and stress of the $\mathrm{Sm}_{4} \mathrm{Co}_{96}$ sample as a function of magnetic field, measured over a range of temperatures. (b) The temperature dependence of the magnetoelastic coupling coefficient $b^{\gamma, 2}$ and the saturation field $H_{\text {sat }}$. Both $b^{\gamma, 2}$ and $H_{\text {sat }}$ increase with decreasing temperature.

Aligning the magnetization with an external magnetic field during growth will result in an atomic displacement due to the magnetoelastic coupling which is locked in by the substrate, thus breaking the symmetry of the amorphous material. To examine whether the imprinted anisotropy has a magnetoelastic origin we can compare the imprinted uniaxial anisotropy energy with the magnetoelastic energy. Assuming an isotropic magnetostriction, the magnetoelastic energy can be written as

$$
E_{\mathrm{me}} \sim-\lambda_{\mathrm{s}} b^{\gamma, 2} \sin ^{2} \theta,
$$

where $\theta$ is the angle between the magnetization and the easy axis [31]. Using the values obtained for the $\mathrm{Sm}_{4} \mathrm{Co}_{96}$ sample we find that $E_{\mathrm{me}} \sim 16 \mathrm{~J} / \mathrm{m}^{3}$ with the magnetization saturated along the hard axis. This is three orders of magnitude smaller than the in-plane uniaxial anisotropy energy $K_{\mathrm{ip}}=$ $5 \times 10^{4} \mathrm{~J} / \mathrm{m}^{3}$, showing that the imprinted anisotropy cannot be magnetoelastic in origin. Instead, magnetic-field induced local configuration changes are the most likely candidate for the anisotropy.

Equating the imprinted anisotropy energy and the magnetoelastic energy we can estimate the stress required for the stress anisotropy (due to the inverse magnetostrictive effect) to exceed the imprinted anisotropy. For the $\mathrm{Sm}_{4} \mathrm{Co}_{96}$ sample we find that this critical stress is $\sigma_{\mathrm{c}}=2 K_{\text {ip }} / 3 \lambda_{\mathrm{s}} \approx 2$ $\mathrm{GPa}$. Figure 4 shows the magnetic response of the film while applying a stress by mechanically bending the sample. The stress thus induced is tensile along the measurement axis and should make the alignment of magnetization along this axis less energetically favorable, due to the negative magnetostriction. Figure 4(a) shows representative hysteresis loops when applying stress along the easy magnetic axis. No significant change is seen in the overall shape of the curves, indicating that the imprinted anisotropy is robust even under stress. However, a small increase is seen in the coercive field, shown in Fig. 4(b), in the 3-6-GPa range. This is consistent with an increase in the anisotropy and is similar to the stress-induced changes in anisotropy observed in $\mathrm{SmCo} / \mathrm{BaTiO}_{3}$ multiferroic heterostructures [33]. Figures 4(c) and 4(d) show the hard axis response and the corresponding saturation field, respectively. Again, the overall shape of the magnetic response does not change significantly and only a small increase in the coercive field is seen (not shown). We do not observe a trend in the saturation field with increasing stress [Fig. 4(d)], even though such changes have been observed in the $\mathrm{SmCo} / \mathrm{BaTiO}_{3}$ heterostructures.

The relatively minor changes in magnetic anisotropy observed when applying large stresses can be explained in terms of the complex relationship between local and global strain in an amorphous alloy system. The magnetostriction is primarily attributed to the highly anisotropic $4 f$ charge distribution of the rare-earth ion, which is coupled to the magnetic moment through the spin-orbit coupling [34]. The rotation of the magnetic moment causes a local strain field, which results in a macroscopic change in shape of the material. In our samples it is the samarium ion which is the main source of the magnetostriction and it is diluted in a cobalt matrix. The precise form of the local strain field will be dependent on the local atomic configuration as the changes in bond lengths are different for the $\mathrm{Sm}-\mathrm{Sm}, \mathrm{Sm}-\mathrm{Co}$, and $\mathrm{Co}-\mathrm{Co}$ bonds [34]. The atomic configuration is not known in the case of amorphous SmCo but can in general be composed of several different types of interconnected atomic clusters, possessing both shortand medium-range order [6,7]. Conversely, the stress induced by mechanical bending is uniform over the entire sample and to first approximation all bonds will be equally stressed. Therefore the local strain fields at the atomic level will not be identical for externally applied stress and magnetostriction. As a result, much larger stress may be required to induce an anisotropy than estimated based on the magnetoelastic energy.

The different compositional dependence of the magnetostriction and anisotropy may also be linked to the local configuration. In three dimensions the bonding percolation limit ranges from 11 to $20 \%$ for lattices with 8 or 12 coordination number $[35,36]$. This means that below the percolation limit the Sm atoms have mostly Co as nearest neighbors and the $\mathrm{Sm}$ can be considered as a perturbation to the Co. Above percolation, a connected network of Sm atoms exists in the material which evidently results in a reduction in the anisotropy but not the magnetostriction. This indicates that the magnetostriction is a single-ion effect with its origin in the Sm ion whereas the anisotropy is related to the coupling between $\mathrm{Sm}$ and Co. 

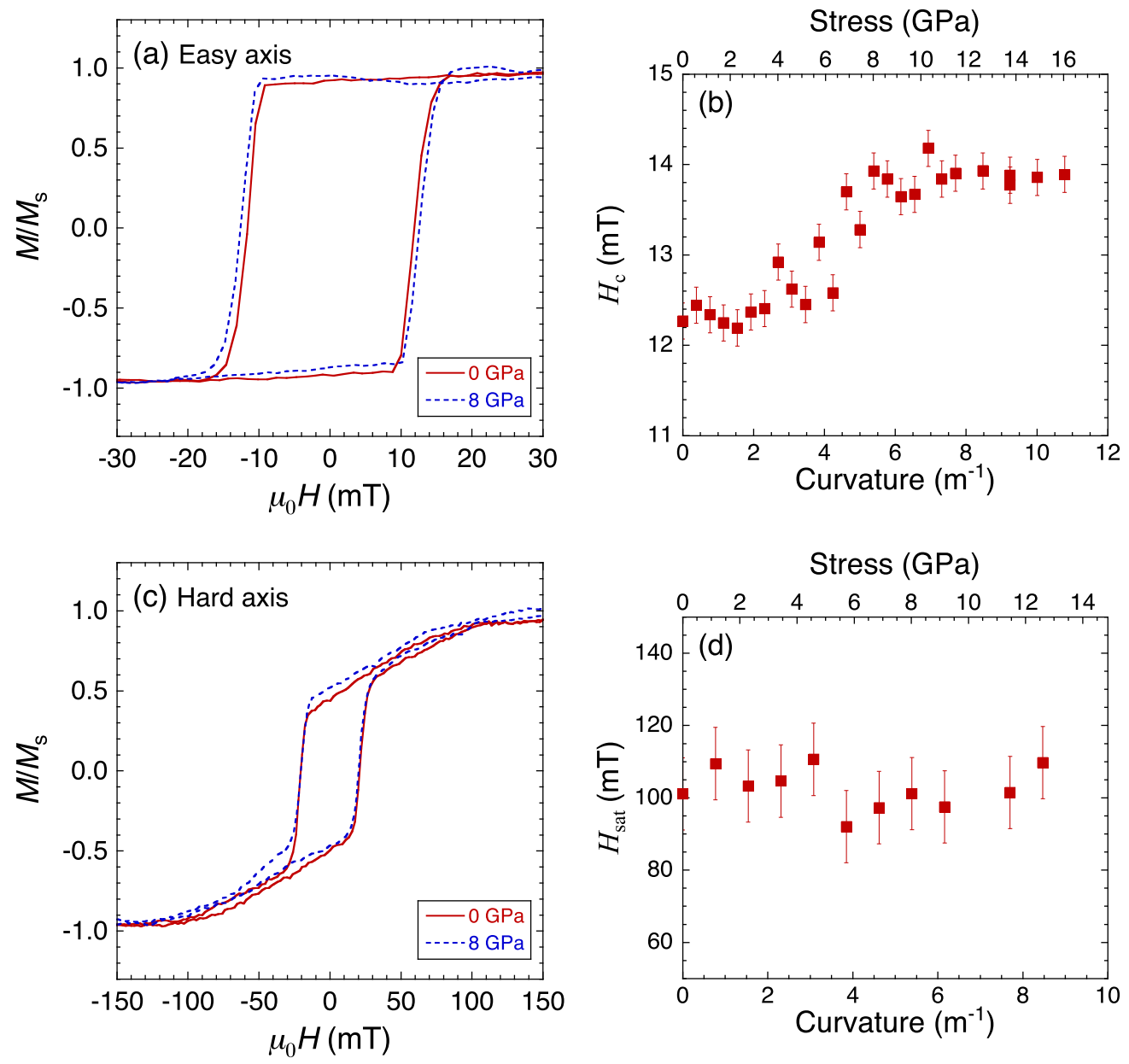

FIG. 4. (Color online) Magnetic response of the $\mathrm{Sm}_{4} \mathrm{Co}_{96}$ film under a mechanically applied stress. (a) The magnetization $M$ along the easy axis, normalized by the saturation magnetization $M_{\mathrm{s}}$, for 0 and $8 \mathrm{GPa}$ of applied stress. (b) The coercive field $H_{\mathrm{c}}$ along the easy axis as a function of stress. (c) The magnetization along the hard axis for 0 and $8 \mathrm{GPa}$ of applied stress. (d) The saturation field $H_{\text {sat }}$ along the hard axis as a function of applied stress.

\section{CONCLUSIONS}

We have used two-dimensional curvature measurements, performed in an applied magnetic field, to determine the magnetostrictive response of amorphous SmCo thin films parallel and perpendicular to the imprinted anisotropy axis. The magnetostriction increases with Sm content but is in all cases rather small compared to other rare-earth-transition-metal compounds, despite the large imprinted magnetic anisotropy. As the composition dependence of the magnetostriction is not the same as that of the anisotropy the two can be tuned independently. The temperature dependences of the magnetostriction and anisotropy are similar, with both increasing as the temperature is reduced. However, comparison of the magnetoelastic and imprinted anisotropy energies shows that the imprinted anisotropy is much larger than the stress anisotropy. The mechanism by which the anisotropy is imprinted can therefore not be only magnetoelastic in nature and we must look elsewhere for the short- or mediumrange order which breaks the symmetry in these amorphous alloys. Only slight changes in anisotropy are detected during the application of large in-plane stresses, which indicates that the global strain thus induced is not equivalent to the local strain involved in magnetostriction. The local strain will depend on the local atomic configurations, which still remain unknown in most amorphous metallic alloys. It is clear that more detailed knowledge about the short- and medium-range order is needed to elucidate effects such as magnetostriction and anisotropy in amorphous materials.

\section{ACKNOWLEDGMENTS}

This work was funded by the Swedish research council (VR), the Knut and Alice Wallenberg foundation (KAW), and the Carl Trygger Foundation.
[1] H. G. Cho, Y. K. Kim, and S.-R. Lee, J. Appl. Phys. 91, 8581 (2002); C.-M. Choi, J.-O. Song, and S.-R. Lee, IEEE Trans. Magn. 41, 2667 (2005).
[2] P. T. Korelis, A. Liebig, M. Björck, B. Hjörvarsson, H. Lidbaum, K. Leifer, and A. R. Wildes, Thin Solid Films 519, 404 (2010). 
[3] D. D. Djayaprawira, K. Tsunekawa, M. Nagai, H. Maehara, S. Yamagata, N. Watanabe, S. Yuasa, Y. Suzuki, and K. Ando, Appl. Phys. Lett. 86, 092502 (2005).

[4] H. Raanaei, H. Nguyen, G. Andersson, H. Lidbaum, P. Korelis, K. Leifer, and B. Hjörvarsson, J. Appl. Phys. 106023918 (2009).

[5] G. Suran, M. Naili, and J. Sztern, J. Appl. Phys. 63, 4318 (1988); G. Suran, M. Naili, H. Niedoba, F. Machizaud, O. Acher, and D. Pain, J. Magn. Magn. Mater. 192, 443 (1999).

[6] A. Hirata, P. Guan, T. Fujita, Y. Hirotsu, A. Inoue, A. R. Yavari, T. Sakurai, and M. Chen, Nat. Mater. 10, 28 (2010).

[7] H. W. Sheng, W. K. Luo, F. M. Alamgir, J. M. Bai, and E. Ma, Nature (London) 439, 419 (2006).

[8] S. Chikazumi, Physics of Ferromagnetism, 2nd ed. (Oxford University, Oxford, 1997).

[9] G. Suran, F. Machizaud, and M. Naili, Phys. Rev. B 47, 15007 (1993).

[10] V. G. Harris, K. D. Aylesworth, B. N. Das, W. T. Elam, and N. C. Koon, Phys. Rev. Lett. 69, 1939 (1992).

[11] N. B. Ekreem, A. G. Olabi, T. Prescott, A. Rafferty, and M. S. J. Hashmi, J. Mater. Process. Tech. 191, 96 (2007).

[12] D. Hunter, W. Osborn, K. Wang, N. Kazantseva, J. HattrickSimpers, R. Suchoski, R. Takahashi, M. L. Young, A. Mehta, L. A. Bendersky, S. E. Lofland, M. Wuttig, and I. Takeuchi, Nat. Commun. 2, 518 (2011).

[13] C. Bormio-Nunes, A. R. Belarmino, C. T. Santos, V. C. Ugeda, C. A. Baldan, and L. Ghivelder, J. Phys. D 42, 165006 (2009).

[14] H. Fujimori, N. S. Kazama, K. Hirose, J. Zhang, H. Morita, I. Sato, and H. Sugawara, J. Appl. Phys. 55, 1769 (1984).

[15] H. Szymczak, J. Magn. Magn. Mater. 200, 425 (1999).

[16] F. Schatz, M. Hirscher, M. Schnell, G. Flik, and H. Kronmüller, J. Appl. Phys. 76, 5380 (1994).

[17] N. H. Duc, K. Mackay, J. Betz, and D. Givord, J. Appl. Phys. 79, 973 (1996).

[18] P. C. Dent, J. Appl. Phys. 111, 07 A721 (2012); K. J. Strnat and R. M. W. Strnat, J. Magn. Magn. Mater. 100, 38 (1991).

[19] D. A. Doane, J. Appl. Phys. 48, 2062 (1977).
[20] F. Magnus, R. Moubah, A. H. Roos, A. Kruk, V. Kapaklis, T. Hase, B. Hjörvarsson, and G. Andersson, Appl. Phys. Lett. 102, 162402 (2013).

[21] M. Gronau, H. Goeke, D. Schuffler, and S. Sprenger, IEEE Trans. Magn. 19, 1653 (1983).

[22] T. Numata, H. Kiriyama, S. Inokuchi, and Y. Sakurai, J. Appl. Phys. 64, 5501 (1988).

[23] K. Chen, H. Hegde, and F. J. Cadieu, Appl. Phys. Lett. 61, 1861 (1992).

[24] E. du Trémolet de Lacheisserie and J. C. Peuzin, J. Magn. Magn. Mater. 136, 189 (1994).

[25] J. P. Jay, F. Petit, J. Ben Youssef, M. V. Indenbom, A. Thiaville, and J. Miltat, J. Appl. Phys. 99, 093910 (2006).

[26] J. P. Jay, F. Le Berre, and S. P. Pogossian, J. Magn. Magn. Mater. 322, 2203 (2010).

[27] M. A. Hopcroft, W. D. Nix, and T. W. Kenny, J. Microelectromech. S. 19, 229 (2010).

[28] L. Sandlund, M. Fahlander, T. Cedell, A. E. Clark, J. B. Restorff, and M. Wun-Fogle, J. Appl. Phys. 75, 5656 (1994).

[29] R. Moubah, F. Magnus, V. Kapaklis, B. Hjörvarsson, and G. Andersson, Appl. Phys. Express 6, 053004 (2013).

[30] F. Magnus, R. Moubah, U. B. Arnalds, V. Kapaklis, A. Brunner, R. Schäfer, G. Andersson, and B. Hjörvarsson, Giant magnetic domains in amorphous SmCo thin films (unpublished).

[31] B. D. Cullity and C. D. Graham, Introduction to Magnetic Materials, 2nd ed. (Wiley, Hoboken, 2009).

[32] J. Betz, K. Mackay, and D. Givord, J. Magn. Magn. Mater. 207, 180 (1999).

[33] R. Moubah, F. Magnus, B. Hjörvarsson, and G. Andersson, J. Appl. Phys. 115, 053905 (2014).

[34] S. Pascarelli, M. P. Ruffoni, A. Trapananti, O. Mathon, C. Detlefs, M. Pasquale, A. Magni, C. P. Sasso, F. Celegato, E. Olivetti, Y. Joly, and D. Givord, Phys. Rev. B 81, 020406 (2010).

[35] C. D. Lorenz, R. May, and R. M. Ziff, J. Stat. Phys. 98, 961 (2000).

[36] C. D. Lorenz and R. M. Ziff, Phys. Rev. E 57, 230 (1998). 